\title{
Decontamination of organic pollutants from aqueous media using polymer-free bioinspired materials
}

Marc Sicard, ${ }^{\dagger}$ Sy/vie Crauste-Manciet, ${ }^{\dagger}$ Francois Dole,$\neq$ Julien Verget, ${ }^{\dagger}$ Alain

Thiérytt and Philippe Barthélémyt,*

tInserm, U1212, Bordeaux, F-33076 France, Université de Bordeaux, F-33076,

Bordeaux, France. UMR CNRS CNRS 5320

¥CNRS, CRPP, UPR 8641, F-33600 Pessac, France

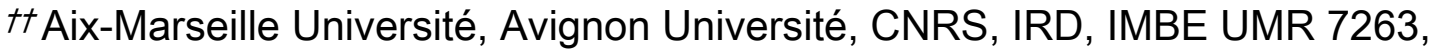

Biomarqueurs, Environnement, Santé (BES), F-13000 Marseille, France.

Number of Pages : 8

Number of Figures : 8

Number of Tables : 3

\section{A/ Equations}

$$
\begin{gathered}
{[\text { Propranolol }] \text { experimental }=\frac{A \text { mixture } 266 \mathrm{~nm} \times \epsilon \text { DiC16dT 289 } \mathrm{nm}-\epsilon \text { DiC16dT 266 } \mathrm{nm} \times A \text { mixture } 289 \mathrm{~nm}}{\epsilon \text { Propranolol } 266 \mathrm{~nm} \times \epsilon \text { DiC16dT } 289 \mathrm{~nm}-\epsilon \text { DiC16dT } 266 \mathrm{~nm} \times \epsilon \text { Propranolol } 289 \mathrm{~nm}}} \\
\% \text { decontamination }=100-\left(\frac{\left\lceil\text { Propranolol }\left.\right|_{\text {experimental }}\right.}{\lceil\text { Propranolol }\rceil_{\text {work solution }}} \times 100\right)
\end{gathered}
$$

Equation 1: Determination of real Propranolol concentration and decontamination percentage by Multicomponent mode method. 
$[$ Diclofenac $]$ experimental $=\frac{\text { A mixture 258nm } \times \in \text { DOTAU } 276 \mathrm{~nm}-\epsilon \text { DOTAU } 258 \mathrm{~nm} \times A \text { mixture } 276 \mathrm{~nm}}{\epsilon \text { Diclofenac } 258 \mathrm{~nm} \times \epsilon \text { DOTAU } 276 \mathrm{~nm}-\epsilon \text { DOTAU } 258 \mathrm{~nm} \times \epsilon_{\text {Diclofenac } 276 \mathrm{~nm}}}$

$\%$ decontamination $=100-\left(\frac{[\text { Diclofenac }\rceil_{\text {experimental }}}{[\text { Diclofenac }]_{\text {work solution }}} \times 100\right)$

Equation 2: Determination of Diclofenac concentration and decontamination percentage by Multicomponent mode method.

\section{B/ Results}

Multicomponent mode method:

Propranolol and Diclofenac concentrations used in this work correspond to absorbances, which did not exceed 1 . The concentrations used were $50 \mu \mathrm{g} . \mathrm{mL}^{-1}$ $(289 \mathrm{~nm})$ for Propranonol and $20 \mu \mathrm{g} \cdot \mathrm{mL}^{-1}(276 \mathrm{~nm})$ for Diclofenac. As shown in Figure ESI 1 the $\lambda$ max of drugs were slightly shifted in the presence of nucleolipids. Also, a shift was observed when the ratio Drugs/Nucleolipids was modified. In these conditions, since a determination of the drugs concentration by the simple Lambert-Beer law at these wavelengths was not possible, a multicomponent mode method was used (Equations 1 and 2). Thus, to determine the drug concentrations in a mixture, epsilon of each compounds (drugs and nucleolipids) at each wavelength (289 nm, $276 \mathrm{~nm}, 266 \mathrm{~nm}$, $258 \mathrm{~nm}$ ) were determined (Table ESI1).

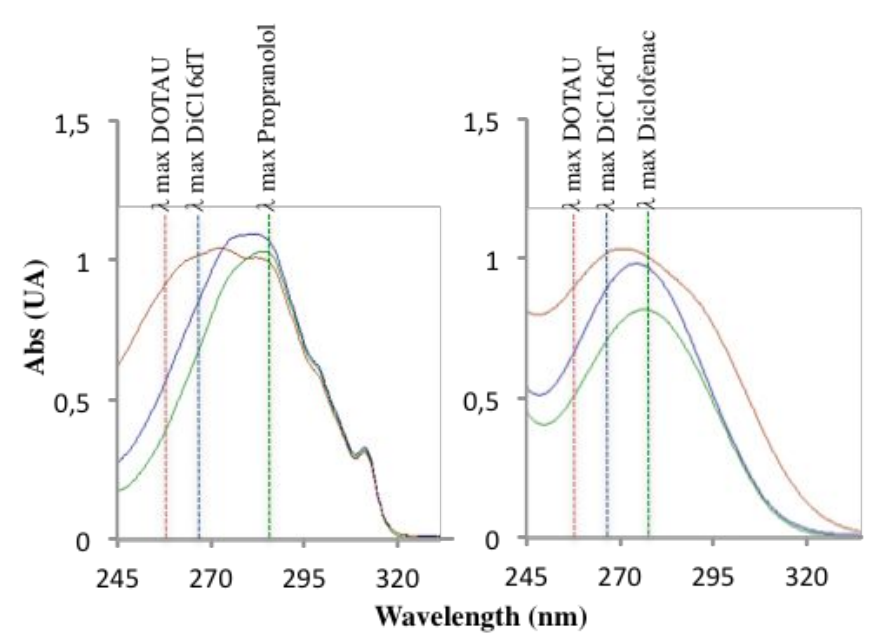

Figure ESI1: Spectra measurement of the Propranolol only $\left(50 \mu \mathrm{g} \cdot \mathrm{mL}^{-1}\right)$, Propranolol and DiC16dT, Propranolol and DOTAU (green, blue and red respectively), Diclofenac only $\left(20 \mu \mathrm{g} \cdot \mathrm{mL}^{-1}\right)$, Diclofenac and DiC16dT, Diclofenac and DOTAU (green, blue and red respectively). 


\begin{tabular}{|c|c|c|c|c|}
\hline & $\mathbf{2 5 8} \mathbf{n m}$ & $\mathbf{2 6 6} \mathbf{n m}$ & $\mathbf{2 7 6} \mathbf{n m}$ & $\mathbf{2 8 9} \mathbf{n m}$ \\
\hline Propranolol & 0,0052 & 0,0089 & 0,1421 & 0,0202 \\
\hline Diclofenac & 0,0219 & 0,0285 & 0,0395 & 0,0251 \\
\hline DiC16dT & 0,0063 & 0,0072 & 0,0058 & 0,0021 \\
\hline DOTAU & 0,0107 & 0,0093 & 0,0049 & 0,0014 \\
\hline
\end{tabular}

Table ESI1: Extinction coefficient $\left(\mathrm{cm}^{-1} \cdot \mu \mathrm{g}^{-1} \cdot \mathrm{mL}\right)$ for the drugs and nucleolipds at differents wavelength $(\lambda \max )$.

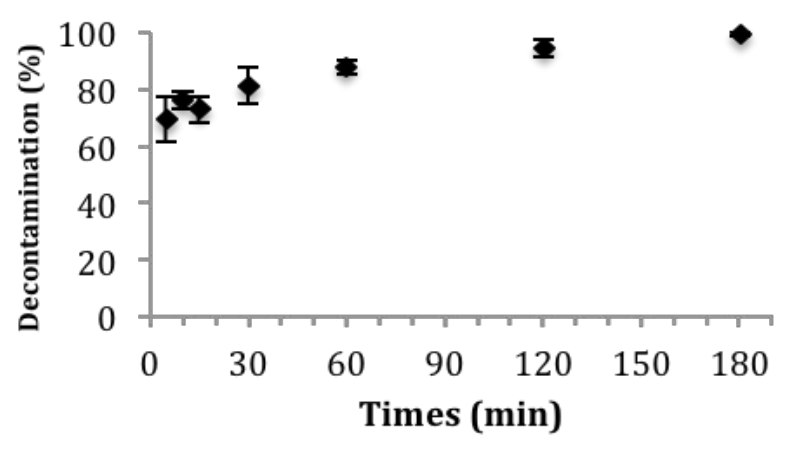

Figure ESI2: Propranolol decontamination kinetics by DiC16dT after filtration $(0.22 \mu \mathrm{m}$ Cellulose) in tap water.

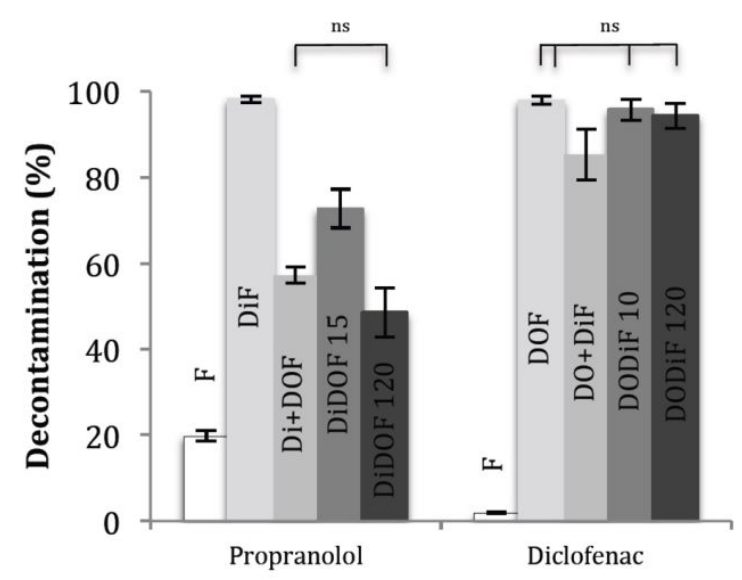

Figure ESI3. Left) Propranolol decontamination in deionized water by filtration only $0.22 \mu \mathrm{m}(\mathrm{F})$, by the DiC16dT and filtration (DiF), by DiC16dT incubation $15 \mathrm{~min}$ followed by DOTAU incubation 60 min (Di+DOF), or by a mixture of DiC16dT and DOTAU with incubation for $15 \mathrm{~min}$ (DiDOF15) or $120 \mathrm{~min}$ (DiDOF120). Right) Diclofenac decontamination in deionised water by filtration only $0.22 \mu \mathrm{m}(\mathrm{F})$, by the DOTAU and filtration (DOF), by DOTAU incubation 10 min followed by DiC16dT incubation $60 \mathrm{~min}$ (DO+DiF), or by a mixture of DOTAU and DiC16dT with incubation times of $10 \mathrm{~min}$ (DODiF10) or $120 \mathrm{~min}$ (DODiF120). (ns means not significant, other comparison are Highly significant, $p<0.005$ ).

\section{LCMS Results}


The LCMS analysis were realized by the Profilomic company. ${ }^{1}$ Results are presented in the tables below. For each molecule are presented: a) $\mathrm{m} / \mathrm{z}, \mathrm{b}$ ) retention time RT, c) linear regression coefficient of the standard range $r 2$, d) the concentrations found in each sample (ND1 = solution not decontaminated; D1, D2, D3 = decontaminated solution, replicates $\mathrm{n}^{\circ} 1,2$ and 3). A "ND" concentration (not detected, table ESI2) means that the molecule is either absent in the sample, or is present at a concentration below the limit of detection (LOD) indicated in the Table ESI3.

\begin{tabular}{|c|c|c|c|c|c|c|c|c|}
\hline \multirow{2}{*}{ Composé } & \multirow{2}{*}{$\mathrm{m} / \mathrm{z}$} & \multirow{2}{*}{ RT (min) } & \multirow{2}{*}{$\begin{array}{c}\mathbf{r}^{2} \text { gamme } \\
\text { étalon }\end{array}$} & \multicolumn{5}{|c|}{ Concentrations ( $\mathrm{\mu g} / \mathrm{L}$ ) } \\
\hline & & & & ND1 théorique & ND1 mesurée & D1 & D2 & D3 \\
\hline metoprolol & 268.19070 & 6.3 & 0.998 & 0.100 & 0.104 & 0.001 & 0.002 & 0.002 \\
\hline propranolol & 260.16450 & 7.99 & 0.997 & 0.100 & 0.085 & ND & ND & ND \\
\hline erythromicine & 734.46800 & 9.23 & 0.999 & 5.000 & 3.998 & ND & ND & ND \\
\hline dichlorvos & 220.95317 & 10.07 & 0.998 & 0.100 & 0.094 & 0.058 & 0.056 & 0.055 \\
\hline fluoxetine & 310.14130 & 10.24 & 0.994 & 0.100 & 0.103 & ND & ND & ND \\
\hline carbamazepine & 237.10223 & 10.44 & 0.997 & 0.100 & 0.128 & 0.081 & 0.070 & 0.066 \\
\hline diuron & 233.02420 & 11.77 & 1.000 & 0.100 & 0.098 & 0.026 & 0.022 & 0.020 \\
\hline isoproturon & 207.14918 & 11.8 & 0.993 & 1.000 & 0.925 & 0.507 & 0.442 & 0.419 \\
\hline ethinylestradiol & 279.17430 & 12.8 & 0.998 & 2.000 & 1.912 & 0.035 & 0.015 & 0.019 \\
\hline alachlore & 270.12550 & 14.85 & 1.000 & 0.100 & 0.092 & 0.003 & 0.002 & 0.002 \\
\hline aclonifen & 265.03740 & 15.59 & 0.962 & 5.000 & 3.762 & ND & ND & ND \\
\hline chlorfenvinphos & 358.97681 & 15.42 & 0.959 & 0.100 & 0.075 & ND & ND & ND \\
\hline quinoxifen & 308.00390 & 16.91 & 0.995 & 1.000 & 2.688 & ND & ND & ND \\
\hline
\end{tabular}

Table ESI2: Table showing the concentration of each pollutants before (Theoric and measured ND1) and after decontamination (triplicate D1, D2, D3). Data are: mass spectrometric peak $(\mathrm{m} / \mathrm{z})$; Liquid chromatography retention time (RT); linear regression coefficient of the standard range ( $\mathrm{r} 2)$; the concentrations found in each sample (ND1 = solution not decontaminated; D1, D2, D3 = decontaminated solution, replicates ${ }^{\circ}{ }^{\circ} 1,2$ and 3).

\begin{tabular}{|c|c|c|c|c|c|}
\hline Micropollutants & Therapeutic classes & Before decontamination $(\mu \mathrm{g} / \mathrm{L}$ ) & After decontamination ( $\mu \mathrm{g} / \mathrm{L}$ ) & Decontamination (\%) & Standard deviation $(\%)$ \\
\hline \multirow{2}{*}{$\begin{array}{l}\text { Carbamazepine } \\
\text { Erythromicine }\end{array}$} & Anticonvulsant & 0.128 & 0.072 & 43.49 & 6.07 \\
\hline & Antibiotic & 3.998 & $0(<L O D)$ & 100 & 0 \\
\hline Ethinylestradiol & Endocrine disruptor & 1.912 & 0.023 & 98.80 & 0.55 \\
\hline Fluoxetine & Antidepressant & 0.103 & $0(<L O D)$ & 100 & 0 \\
\hline Metoprolol & Beta blocker & 0.104 & 0.0017 & 98.40 & 0.56 \\
\hline Propranolol & Beta blocker & 0.085 & $0(<L O D)$ & 100 & 0 \\
\hline Aclonifen & Pesticide & 3.762 & $0(<\mathrm{LOD})$ & 100 & 0 \\
\hline Alachlore & Pesticide & 0.092 & 0.0023 & 97.46 & 0.63 \\
\hline Chlorfenvinphos & Pesticide & 0.075 & $0(<L O D)$ & 100 & 0 \\
\hline Dichlorvos & Pesticide & 0.094 & 0.056 & 40.07 & 1.63 \\
\hline Diuron & Pesticide & 0.098 & 0.023 & 76.87 & 3.12 \\
\hline Isoproturon & Pesticide & 0.993 & 0.46 & 54.08 & 4.60 \\
\hline Quinoxifen & Pesticide & 2.688 & 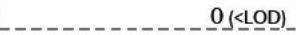 & 100 & -0 \\
\hline & tail & 14.132 & 0.63 & 95.51 & 0.48 \\
\hline
\end{tabular}

Table ESI3: Table showing the concentration of each pollutants before and after decontamination, the percentage and standard deviation. LOD means Limit Of Detection.

\footnotetext{
${ }^{1}$ Report Profilomic (http://www.profilomic.com/en/), Bulletin d'analyse de 4 échantillons d'eau avant et après décontamination, ETUDE 111-PFM-001.
} 

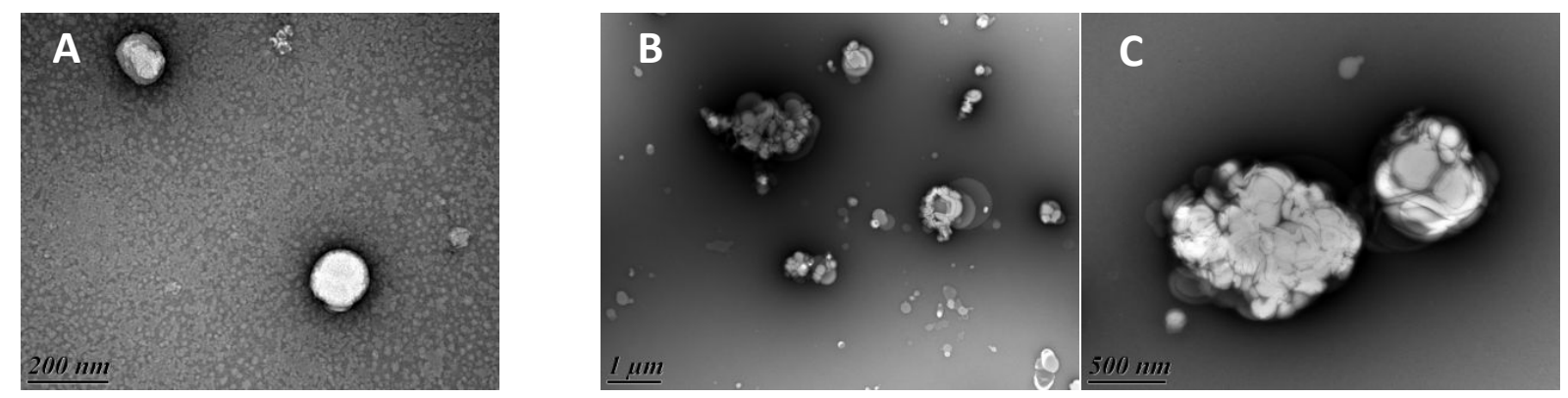

Figure ESI4: TEM images (Transmission Electronic Microscopy) showing: A) aggregates of 120 $\mathrm{nm}$ in diameter (diC16dT), B) and C) larger aggregates of $0.5-2 \mu \mathrm{m}$ after addition of diC16dT to solutions contaminated with Diclofenac. TEM microscopy experiments were performed with a Hitachi H 7650 (negative staining with Uranyle acetate $2.5 \%$ in water, Ni carbon coated grids). 
Figure ESI5: Absorbance calibration curves, DiC16dT
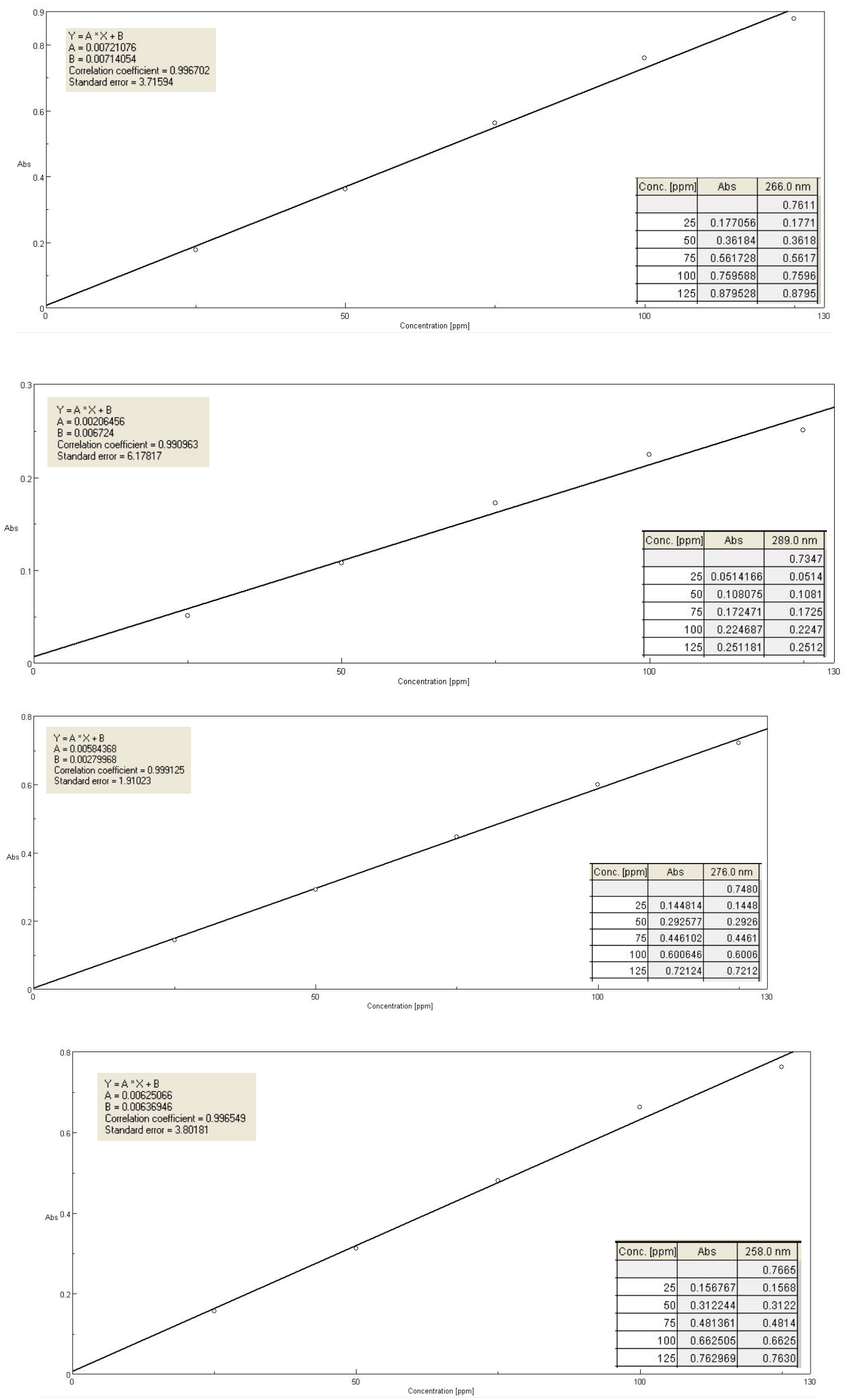
Figure ESI6: Absorbance calibration curves, DOTAU
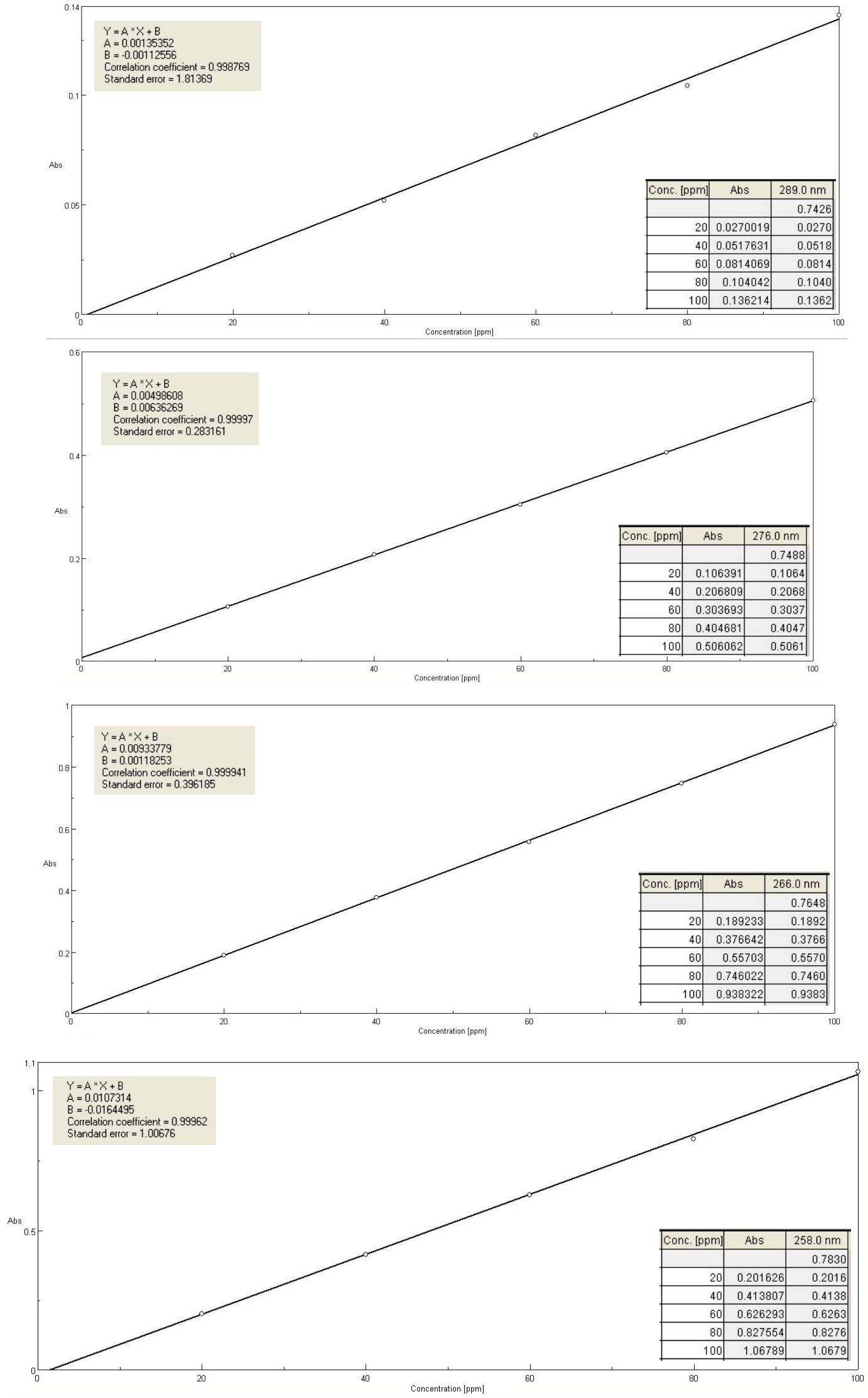
Figure ESI7: Absorbance calibration curves, Diclofenac
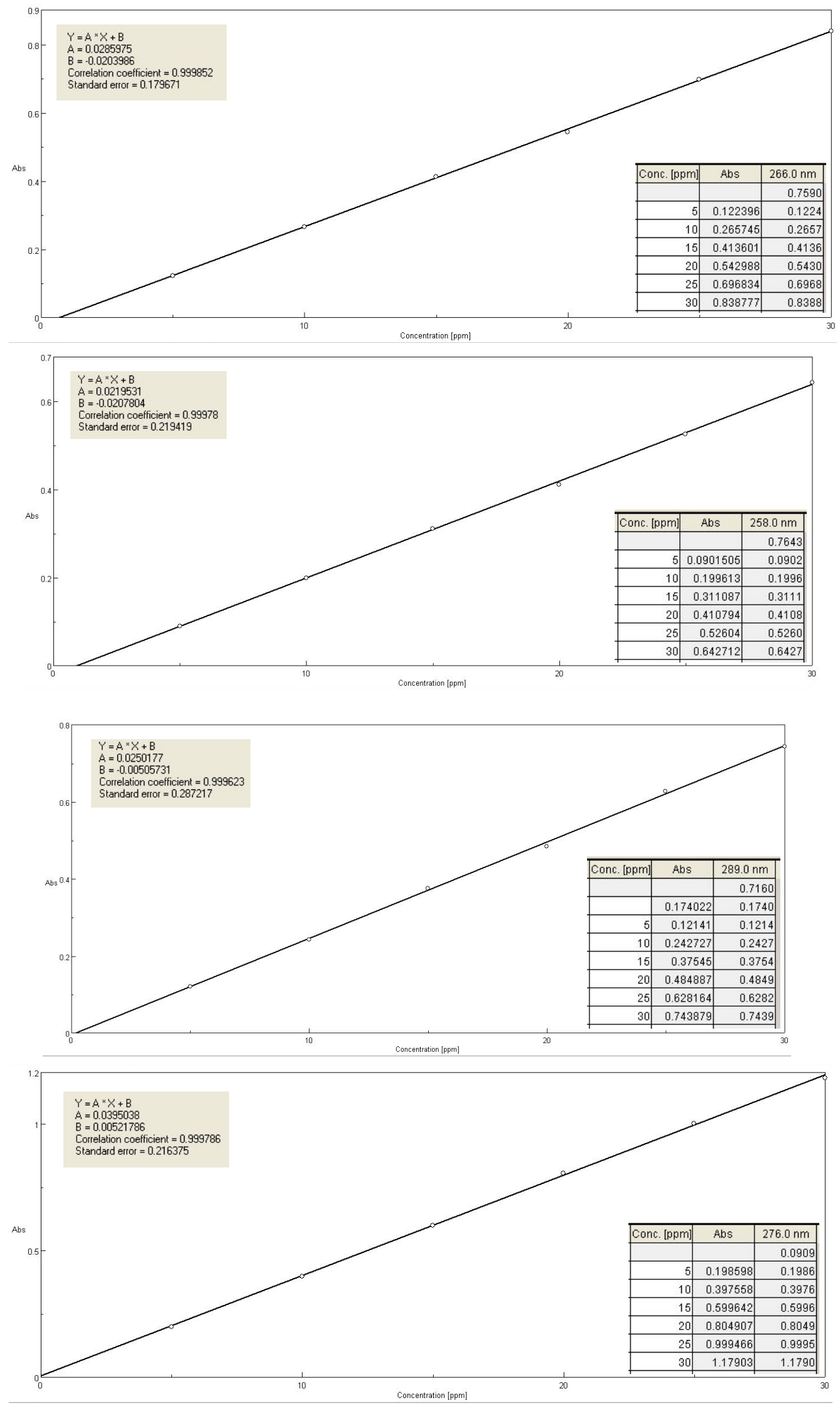
Figure ESI8: Absorbance calibration curves, Propranolol
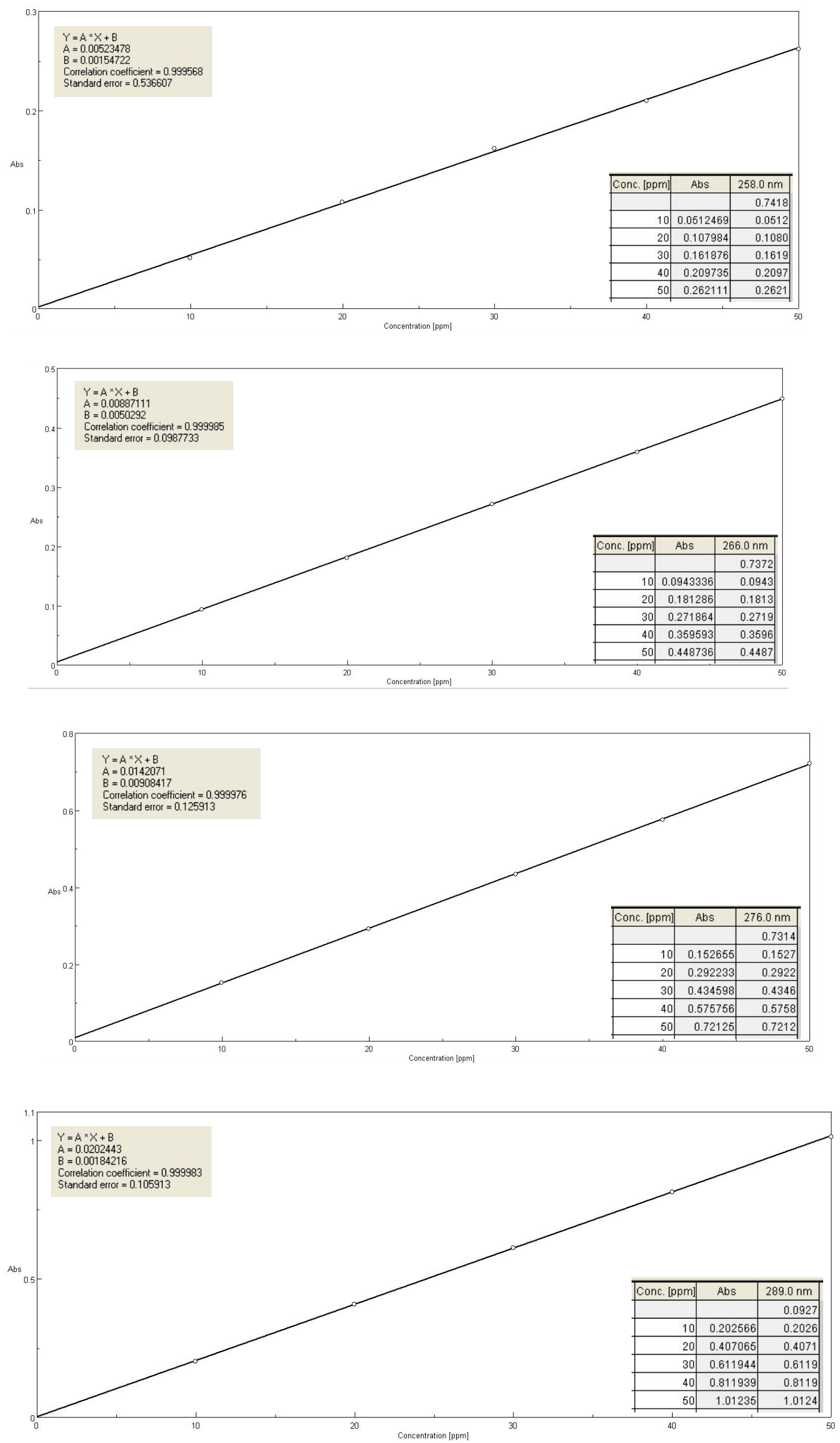